\title{
Hanbury-Brown-Twiss interferometry for sonoluminescence bubble
}

\author{
Y. Hama, ${ }^{1}$ T. Kodama, ${ }^{2}$ and Sandra S. Padula ${ }^{3}$ \\ ${ }^{1}$ Instituto de Física, Universidade de São Paulo, Caixa Postal 66318, 05315-970 São Paulo, Brazil \\ ${ }^{2}$ Instituto de Física, Universidade Federal do Rio de Janeiro, Caixa Postal 68.528, 21945-970 Rio de Janeiro, Brazil \\ ${ }^{3}$ Instituto de Física Teórica, UNESP, Rua Pamplona 145, 01405-901 São Paulo-SP, Brazil
}

(Received 30 December 1996; revised manuscript received 22 April 1997)

\begin{abstract}
Two-photon correlation of the light pulse emitted from a sonoluminescence bubble is discussed. It is shown that several important features of the mechanism of light emission, such as the time scale and the shape of the emission region, could be obtained from Hanbury-Brown-Twiss interferometry. We also argue that such a measurement may serve to reject one of the two currently suggested emission mechanisms, i.e., the thermal process versus the dynamical Casimir effect.
\end{abstract}

[S1050-2947(97)06008-3]

PACS number(s): 42.50.Ar, 78.60.Mq, 43.35.+d

The sonoluminescence process converts the acoustic energy in a fluid medium into a short light pulse emitted from the interior of a collapsing small cavitating gas bubble. Since the discovery of the technique for trapping a single cavitating bubble by a standing acoustic wave $[1,2]$, many remarkable properties have been revealed [3-6]. The spectrum of emitted light is very wide, extending from the visible to the ultraviolet regions. The conversion process requires some mechanism of extraordinary concentration of the energy and is apparently related to keeping the sphericity of the collapsing bubble while its radius shrinks more than one order of magnitude. An important fact is that the light emission takes place within a very short period of time compared to the typical scale of hydrodynamic motion of the bubble. The sensitivity of the radiation power and spectrum to physical parameters, such as temperature, pressure, the amplitude of acoustic drive, and the gas composition, is the most intriguing aspect of the process and no apparent reason nor any well-defined systematics are known. In particular, the emission mechanism of light is still controversial. Some authors attribute the light emission to the quantum-electrodynamic vacuum property based on the dynamical Casimir effect [7-9]. Others consider that thermal processes [10-12] such as black-body radiation should be the natural explanation for the process. Nonequilibrium atomic collision processes could also be a strong candidate [13]. In any case, the gas dynamics inside the bubble [14,15], in particular, the shock wave formation, seems to play an essential role [16]. However, experimental information on the dynamics of the gas inside the bubble is not available at present. Due to the short time scale and to the smallness of the emission region, precise measurement of this geometric and dynamical information is quite difficult. Usually, the time dependence of the bubble radius is measured using the Mie scattering process of laser beams $[3,17]$. Nevertheless, since we have no information on the properties of the gas during the implosion phase, it is not obvious that the scattered laser amplitude is really measuring the bubble surface. On the other hand, in addition to the knowledge of time elapsed in the process, it would also be desirable to determine the shape of the emission region.

The two-photon interferometry, initially proposed by Hanbury-Brown and Twiss (HBT) [18] for measuring stellar sizes, later found its application in the analysis of highenergy particle production processes [19] and is now widely employed in relativistic heavy-ion collisions [20,21]. The basic principle is associated with the Bose-Einstein statistics obeyed by the identical particles involved in the process, and to the chaoticity of the emission mechanism. If the emitting source has no additional dynamical correlation, then the twoparticle correlation is directly related to the geometrical size of the source. Several authors [22,23] have discussed the effect of the source dynamics on pion interferometry. In this paper, we apply this well-established method to obtain some important information on the dynamics of the gas inside the bubble while it emits the light pulse. It is interesting to note that the application of HBT interferometry to the sonoluminescence bubble $\left(R \sim 10^{-5} \mathrm{~m}\right)$ lies between the stellar $\left(R \sim 10^{10} \mathrm{~m}\right)$ and the high-energy physics $\left(R \sim 10^{-15} \mathrm{~m}\right)$ scales.

The HBT interferometry method consists of measuring two light quanta in coincidence. Let $P_{2}\left(\vec{k}_{1}, \vec{k}_{2}\right)$ be the probability for simultaneously detecting two photons with wave vectors $\vec{k}_{1}$ and $\vec{k}_{2}$ and $P_{1}\left(\vec{k}_{i}\right)$ the single-photon probability. The correlation function $C\left(\vec{k}_{1}, \vec{k}_{2}\right)$ is then defined as

$$
C\left(\vec{k}_{1}, \vec{k}_{2}\right) \equiv \frac{P_{2}\left(\vec{k}_{1}, \vec{k}_{2}\right)}{P_{1}\left(\vec{k}_{1}\right) P_{1}\left(\vec{k}_{2}\right)} .
$$

If the light is emitted by chaotic, static, and independent elementary sources, whose space-time distribution is given by $\rho(x)$, each one emitting one photon at a time, the correlation function is simply related to the Fourier transform of $\rho(x)$ as ${ }^{1}$

$$
C\left(\vec{k}_{1}, \vec{k}_{2}\right)=1+\frac{1}{2} \frac{|\widetilde{S}(\vec{q}, \vec{K})|^{2}}{\widetilde{S}\left(0, \vec{k}_{1}\right) \widetilde{S}\left(0, \vec{k}_{2}\right)},
$$

where

\footnotetext{
${ }^{1}$ Here, we adopt the notation used in [24], applying it to our case. The factor $1 / 2$, in the second term, comes from the spin 1 character of the photon.
} 
TABLE I. The analytic expressions of the correlation function for some typical parametrization of the source density $\rho(r, t)$ are shown.

\begin{tabular}{lcc}
\hline \hline & Form of the source & $C\left(\vec{k}_{1}, \vec{k}_{2}\right)-1$ \\
\hline Case A & $e^{-r^{2} / 2 R^{2}} e^{-t^{2} / 2 \tau^{2}}$ & $e^{-(\Delta \omega)^{2} \tau^{2}} e^{-q^{2} R^{2} / 2}$ \\
Case B & $\delta(r-R) e^{-t^{2} / 2 \tau^{2}}$ & $e^{-(\Delta \omega)^{2} \tau^{2}[\sin (q R) /(q R)]^{2} / 2}$ \\
Case C & $\Theta(R-r) e^{-t^{2} / 2 \tau^{2}}$ & $9 e^{-(\Delta \omega)^{2} \tau^{2}}\left\{[\cos (q R)-\sin (q R) /(q R)] /(q R)^{2}\right\}^{2} / 2$ \\
Case D & $e^{-r / R} \Theta\left(3 \tau^{2}-t^{2}\right)$ & {$[\sin (\Delta \omega \sqrt{3} \tau) / \Delta \omega \sqrt{3} \tau]^{2}\left(1+q^{2} R^{2}\right)^{-4} / 2$} \\
Case E & $\Theta(\dot{R} t-r) e^{-t^{2} / \tau^{2}} \Theta(t)$ & $9|I|^{2} /\left(8 \mu^{6}\right)$, \\
& & $I=-i \sqrt{\pi}\left[\left(1+\mu z^{+}\right) W\left(z^{+}\right)-\left(1-\mu z^{-}\right) W\left(z^{-}\right)\right]-2 \mu$ \\
\hline \hline
\end{tabular}

$$
\widetilde{S}(\vec{q}, \vec{K})=\int d^{4} x e^{-i q x} \rho(x) j^{*}\left(\vec{k}_{1}\right) j\left(\vec{k}_{2}\right),
$$

$\vec{q}=\vec{k}_{1}-\vec{k}_{2}, \vec{K}=\left(\vec{k}_{1}+\vec{k}_{2}\right) / 2$, and $j\left(\vec{k}_{i}\right)$ is the amplitude for the emission of a photon with the wave vector $\vec{k}_{i}$ at the source point $x$. So, it is possible to determine the source geometry from the measurement of $C\left(\vec{k}_{1}, \vec{k}_{2}\right)$. In general situations, though, the relationship between the correlation function and the source geometry is more complex, not being possible to determine uniquely its geometry only from the knowledge of $C\left(\vec{k}_{1}, \vec{k}_{2}\right)$. Nevertheless, in the absence of phase space correlations, once we have a good guess about the shape and the time development of the emitting source, we could suggest an appropriate parametrization for the geometry of the process, and the HBT measurements can be used to determine these parameters.

It should be emphasized that the chaoticity of the emitting source, which manifests as random phases of an emitted signal, plays a crucial role in relating the correlation function to the geometry of the source. In contrast to this, if the emission process is coherent (for example, a laser source), it is well known $[20,25,26]$ that no HBT correlation would be observed, that is,

$$
C\left(\vec{k}_{1}, \vec{k}_{2}\right) \equiv 1
$$

As an immediate consequence, we can distinguish these two extreme scenarios with a precise HBT measurement. As mentioned before, the mechanisms of light emission proposed so far can be classified into two categories: One based on rather conventional atomic process of thermal origin, and the other due to the dynamical Casimir effect. In the latter case, a coherent burst of light would be produced, whereas for the former scenario we expect chaotic emissions. In both cases the single-photon spectra are similar to that of the black-body radiation, but an HBT analysis would allow for clearly differentiating these two emission mechanisms, shedding some light on the most intriguing feature of the sonoluminescence phenomenon.

Let us now investigate some examples of nontrivial HBT correlation functions $(C \neq 1)$. For this, we assume that light quanta emitted from different space-time points have no extra correlation in addition to that due to the Bose-Einstein statistics. This is the case when, for example, the emission process has its origin in atomic collisional or bremsstrahlung mechanisms. We further assume that the gas inside the bubble is locally in thermal equilibrium. This does not nec- essarily mean that the emitted photons are also in thermal equilibrium with the gas. For simplicity, we only treat here spherically symmetrical sources [6].

Since the time scale of the hydrodynamic motion is considered to be few orders of magnitude greater than the time scale of the emitting process $(\sim 10 \mathrm{ps})$, we first consider that the source size remains constant during the light emission. Thus, the time and space dependences are factorized. In Table I, we show the analytic expressions of the correlation function for some typical parametrization of the source density $\rho(r, t)$. The first three cases $(\mathrm{A}, \mathrm{B}$, and $\mathrm{C})$ refer, respectively, to a Gaussian type of source, to a spherical shell, and to a homogeneous sphere, all of them having a Gaussian lapse of time. The fourth example (D) corresponds to an exponential spatial distribution shining constantly within an interval of time $\tau$.

It could well be possible that the emission region is not static, in which case the time dependence of the source size should be considered. One may, for instance, imagine that the radiating source is the spherical domain behind the expanding shock front formed at the center [11,10]. In any case, the fluid velocity is certainly much smaller than the speed of light so that any dynamical effect due to the fluid motion could be completely neglected. Thus, Eq. (3) is still valid in this case. An emitting source with these characteristics is represented by case $\mathrm{E}$.

In Table I, $q=\left|\vec{k}_{1}-\vec{k}_{2}\right|, \quad \Delta \omega=\omega_{1}-\omega_{2}=c\left(k_{1}-k_{2}\right)$, $\mu=\dot{R} \tau q, z^{ \pm}=(\Delta \omega \pm \dot{R} q) \tau / 2$ and $W(z) \equiv e^{-z^{2}} \operatorname{erfc}(-i z) . R$ is the spatial extension parameter, $\tau$ is the time span parameter of the source, and $\dot{R}$ is the velocity of the shock wave. Case A was studied by Trentalange and Pandey [27], following the prescription given in [28]. However, as pointed out by Slotta and Heinz [24], the current conservation should be correctly taken into account, which results in a slight deviation from Eq. (6) of Ref. [27]. Note that in the first four cases (A-D) above, the correlation functions are written in the form

$$
C\left(\vec{k}_{1}, \vec{k}_{2}\right)=1+\frac{1}{2} T(\Delta \omega) \Phi(q),
$$

which is a consequence of the factorized form of the source. Therefore, to determine the time span parameter $\tau$, it is convenient to plot the data for fixed $q$. If the source is really factorized in space and time, then plots of $\ln (C-1)$ vs $(\Delta \omega)^{2}$ for different pairs of $\left(\vec{k}_{1}, \vec{k}_{2}\right)$ should generate a set of identical curves just shifted from each other for different values of $q=\left|\vec{k}_{1}-\vec{k}_{2}\right|$. In Fig. 1, we show examples of such 


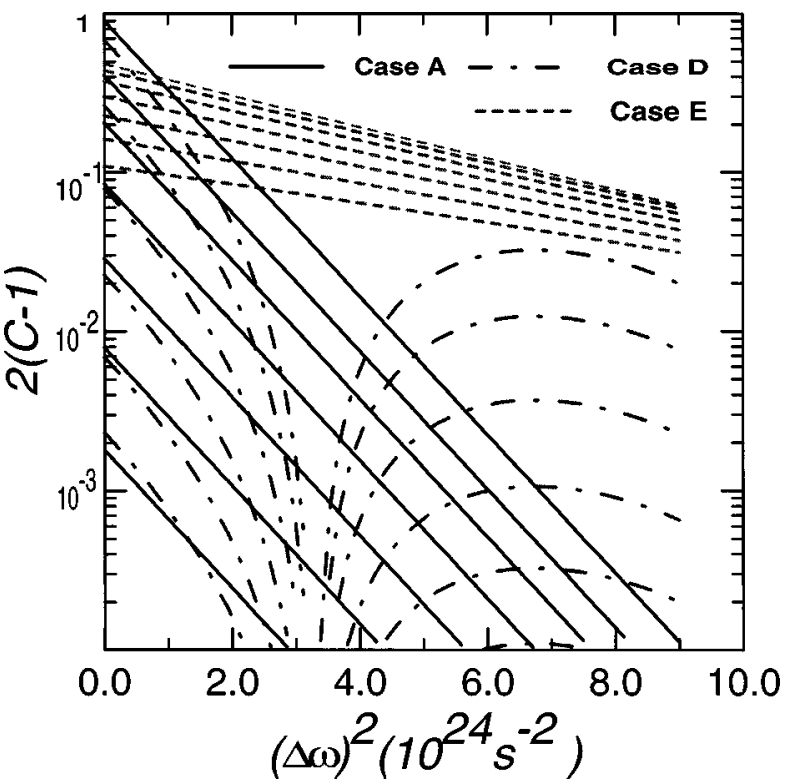

FIG. 1. Correlation functions plotted as functions of $\Delta \omega$ for various values of $q$. The solid lines refer to case A, dashed-dotted lines are for case D and dashed lines are for case E. The first two cases are examples of factorized sources, whereas the lines for case E are not parallel.

plots, with $\tau=1 \mathrm{ps}, R=1 \mu \mathrm{m}$ in the blue-light domain $\left(k_{1} \simeq k_{2} \simeq 1.3 \times 10^{7} \mathrm{~m}^{-1}\right)$. The solid lines refer to case $\mathrm{A}$, the dashed-dotted lines to case $\mathrm{D}$, and the dashed lines to case $\mathrm{E}$. As can be seen in this figure, if the time span is Gaussian and factorized from the space dependence, then these curves should be parallel straight lines whose slopes would give the value of the parameter $\tau$. If the time span is not Gaussian, then the curves are not straight lines, but similar to those for case D. However, even in this case, the first derivatives of the corresponding curves with respect to $(\Delta \omega)^{2}$ at the origin provide an estimate of the parameter $\tau$. The information about the source spatial distribution $\Phi(q)$, is determined by means of the intersection of these lines with the abscissa. For case E of Fig. 1, the velocity of the expansion was taken to be $\dot{R}=2 \times 10^{-4} c$, where $c$ is the speed of light. If the velocity is smaller than this value, the lines corresponding to different $q$ 's come closer to one another. This happens because, for smaller velocities, the effective emission region becomes smaller and, consequently, the correlation function becomes broader and slow varying with $q$. Note that for case $\mathrm{E}$, the lines are not parallel, reflecting the nonfactorized emission source.

In practice, such an analysis might be limited by experimental conditions. In particular, to get a meaningful result for the $\tau$ parameter, $\Delta \omega$ should be measured within a resolution of the order of $1 / \mathrm{ps}$, which corresponds to measuring the photon energy itself within precision of

$$
\frac{\delta \omega}{\omega}<\frac{10^{12} \mathrm{~s}^{-1}}{3.9 \times 10^{15} \mathrm{~s}^{-1}} \simeq 2.5 \times 10^{-4},
$$

for blue light. This might not be easily achieved. However, even if the energy resolution is not high enough, the spatial factor in Eq. (3) can independently be analyzed if the source is factorized. Being so, the HBT correlation function of two

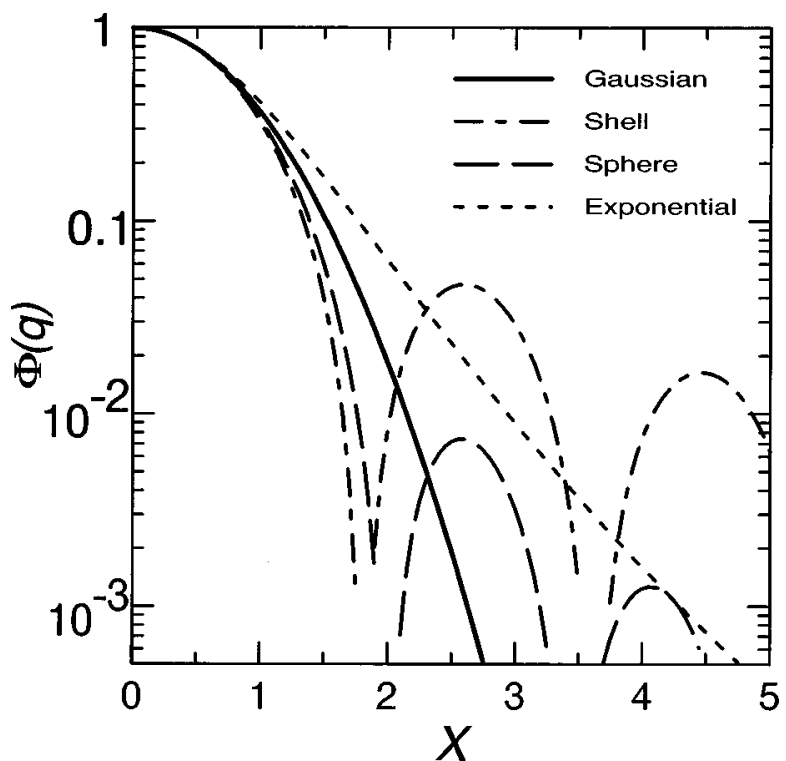

FIG. 2. Geometrical form factor $\Phi(q)$ plotted as functions of $X=\sqrt{-(1 / 2)\left[d^{2} \Phi(0) / d q^{2}\right]} q$. The solid curve corresponds to the Gaussian source, the dashed-dotted lines to the spherical shell, the broken lines to the spherical source, and the dashed lines to the exponential distribution.

photons of approximately the same energy, under a poor energy resolution (within $\delta \omega$ ), would lead to an angular correlation given by

$$
C\left(\vec{k}_{1}, \vec{k}_{2}\right) \rightarrow C\left(\vec{k}_{1}, \vec{k}_{2}\right)=1+1 / 2\langle T(\delta \omega\rangle) \Phi(q) .
$$

Since the multiplicative factor $\langle T(\delta \omega)\rangle$ is independent of $q$, it can easily be eliminated from $C$ near the origin $q=0$. The necessity of the above renormalization of the correlation function at the origin as a consequence of a poor energy resolution of HBT measurements has been pointed out in [22]. The function $\Phi(q)$ in Eq. (3) behaves quadratically in $q$ around $q=0$. Namely,

$$
\Phi(q)=\left\{\begin{array}{cc}
1-R^{2} q^{2}+\cdots, & \text { Gaussian }(\mathrm{A}) \\
1-R^{2} q^{2} / 3+\cdots, & \text { shell }(\mathrm{B}) \\
1-R^{2} q^{2} / 5+\cdots, & \text { sphere }(\mathrm{C}) \\
1-4 R^{2} q^{2}+\cdots, & \text { exponential (D) }
\end{array}\right.
$$

so that we can determine the parameter $R$ by the curvature of $\Phi(q)$ at the origin. By defining

$$
\kappa \equiv-\left.\frac{d^{2} \Phi}{d q^{2}}\right|_{q=0}
$$

we have

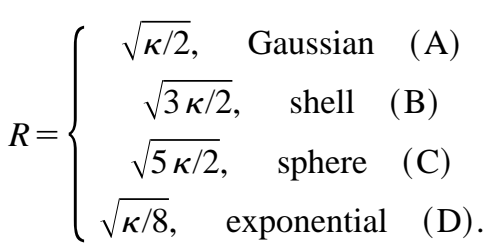


To distinguish among the shapes of the correlation functions reflecting different source density distributions, we would need to know the behavior of $\Phi(q)$ in a wider range in $q$. To stress the differences among the four cases above, let us introduce the variable $X$ defined as

$$
X \equiv \sqrt{\kappa / 2} q .
$$

Then, by definition, $\Phi$ behaves as

$$
\Phi=1-X^{2}+\cdots,
$$

near $X=0$. In Fig. 2, we compare the behavior of $\Phi$ as a function of $X$ for the four cases. The continuous line corresponds to the Gaussian spatial distribution, the broken lines to the sphere, the dashed-dotted ones to the spherical shell, and, finally, the dashed lines correspond to the exponential density distribution. As can be seen in this figure, the differences among these curves are not striking near the origin, but if the data are precise enough ( $\sim 3$ order of magnitudes) in a sufficiently wide range of $X$, we may determine the shape of the source function $\rho$.
As already mentioned, if the emitting process were coherent, the behavior of the correlation function would be entirely different from the ones just discussed. As anticipated in the beginning, a precise measurement of the two-photon correlation function of the light quanta emitted by a sonoluminescence bubble would allow us to distinguish between chaotic and coherent emission mechanisms. However, we should stress that, even in the case of a chaotic source, a poor energy-resolution experiment could lead to a result similar to the one expected in the case of a coherent source, due to the factor $\exp \left(-\tau^{2} \Delta \omega^{2}\right)$. Therefore, a very high energyresolution experiment is required to clearly differentiate between these two opposite scenarios.

This work has been partially supported by FAPESP (Contract No. 95/4635-0), MCT/FINEP/CNPq (PRONEX) under Contract No. 41.96.0886.00 and FAPERJ. One of us, (T.K.) expresses his gratitude to J. Rafelski, I.Scott, and H-T. Elze for stimulating discussions on sonoluminescence phenomena and encouragements. Discussions with C.E. Aguiar, L. M. Pimentel, and P.A. Nussenzweig are gratefully acknowledged.
[1] D.F. Gaitan and L.A. Crum, J. Acoust. Soc. Am. Suppl. 187 , S141 (1990); D.F. Gaitan, L.A. Crum, C.C. Curch, and R.A. Roy, J. Acoust. Soc. Am. 91, 3166 (1992).

[2] L.A. Crum, Phys. Today 47(9), 22 (1994).

[3] B.P. Barber and S. Putterman, Phys. Rev. Lett. 69, 3839 (1992).

[4] B.P. Barber, C.C. Wu, R. Löfstedt, P.H. Roberts, and S. Putterman, Phys. Rev. Lett. 72, 1380 (1994).

[5] R. Heller, K. Weninger, S. Putterman, and B.P. Barber, Science 266, 248 (1994).

[6] K. Weninger, S. Putterman, and B.P. Barber, Phys. Rev. E 54, R2205 (1996).

[7] K.A. Milton, Report No. hep-th/9510091.

[8] C. Eberlein, Phys. Rev. A 53, 2772 (1996).

[9] A. Chodos, Report No. hep-ph/9604368.

[10] R. Löfstedt, B.P. Barber, and S.J. Putterman, Phys. Fluids A 5, 2911 (1993).

[11] W.C. Moss, D.B. Clarke, J.W. White, and D.A. Young, Phys. Lett. A 211, 69 (1996).

[12] Seth Putterman, Sci. Am. (Int. Ed.) 272 (Feb.), 32 (1995).

[13] L. Frommhold and A.A. Atchley, Phys. Rev. Lett. 73, 2883 (1994).

[14] I. Scott, H-Th. Elze, T. Kodama, and J. Rafelski (unpublished).

[15] M.C. Chu, Phys. Rev. Lett. 76, 4632 (1996).

[16] C.C. Wu and P.H. Roberts, Phys. Rev. Lett. 70, 3424 (1993).
[17] W.J. Lentz, A.A. Atchley, and D.F. Gaitan, Appl. Opt. 34, 2648 (1995).

[18] R. Hanbury-Brown and R.Q. Twiss, Philos. Mag. 45, 663 (1954).

[19] G. Goldhaber, S. Goldhaber, W. Lee, and A. Pais, Phys. Rev. 120, 300 (1960).

[20] See, for example, M. Gyulassy, S.K. Kauffmann, and L.W. Wilson, Phys. Rev. D 20, 2267 (1979).

[21] Proceedings of the International Workshop on Correlations and Multiparticle Production (CAMP/LESIP IV), edited by M. Pluemer, S. Raha, and and R. M. Weiner (World Scientific, Singapore, 1991).

[22] Y. Hama and S. S. Padula, Phys. Rev. D 37, 3237 (1988).

[23] S. Pratt, Phys. Rev. D 33, 1314 (1986); K. Kolehmainen and M. Gyulassy, Phys. Lett. B 180, 203 (1986); S. S. Padula and M. Gyulassy, Nucl. Phys. B 339, 378 (1990).

[24] C. Slotta and U. Heinz, Phys. Lett. B 391, 469 (1997).

[25] R.J. Glauber, Quantum Theory of Coherence in Quantum Optics, edited by S.M. Kay and A. Maitland (Academic, New York, 1970).

[26] C-Y. Wong, Introduction to High-Energy Heavy-IonCollisions (World Scientific, Singapore, 1994), p. 431.

[27] S. Trentalange and S.U. Pandey, J. Acoust. Soc. Am. 99, 2439 (1996).

[28] D. Neuhauser, Phys. Lett. B B182, 289 (1986). 